\title{
Enzasataurin Enhances ATRA-induced Differentiation of Acute Myeloid Leukemia Cells
}

\author{
Ze-yi Li \\ Shanghai Jiaotong University School of Medicine affiliated \\ Cui Liang \\ Changhai Hospital, The Second Military Medical University \\ Ming Ding \\ Central Hospital of Minhang District \\ Xiang-qin Weng \\ Ruijin Hospital Affiliated to Shanghai Jiao Tong University School of Medicine \\ yan Sheng \\ Shanghai Jiaotong UNiversity School of Medicine Affiiliated Ruijin Hospital \\ Jing Wu \\ Ruijin Hospital Affiliated to Shanghai Jiao Tong University School of Medicine \\ Hao Lu \\ Shanghai Jiaotong University school of medicine affiliated Ruijin Hospital \\ xun cai ( $\nabla$ xuncai@yahoo.com ) \\ Shanghai Jiaotong University School of Medicine affiliated Ruijjin Hospital https://orcid.org/0000-0003-4372-8889
}

Research

Keywords: acute myeloid leukemia, all-trans retinoic acid, differentiation, enzastaurin

Posted Date: July 9th, 2020

DOI: https://doi.org/10.21203/rs.3.rs-38343/v1

License: (c) (i) This work is licensed under a Creative Commons Attribution 4.0 International License. Read Full License 


\section{Abstract Background}

All-trans retinoic acid (ATRA) is considered to be the sole clinically useful differentiating agent in the treatment of acute myeloid leukemia (AML). However, it has been effective only in acute promyelocytic leukemia (APL) but not other subtypes of AML. Therefore, finding strategies to sensitize cells to ATRA may develop ATRA-based therapy in the treatment of non-APL AML patients.

\section{Methods}

Cell proliferation was assessed by cell growth. Cell death was evaluated by cell viability and Annexin-V assay. Cell differentiation was analyzed by CD11b expression and morphology. To explore the underlying mechanisms, we studied the role of PKC $\beta$, MEK, ERK, AKT, PU.1, C/EBP $\beta$ and C/EBPE by Western-blotting analysis.

\section{Results}

In this study, a clinically achievable concentration of enzastaurin enhanced ATRA-induced differentiation of AML cell lines, HL-60 and U937 as well as non-APL AML primary cells, while it also restored ATRA sensitivity in ATRA-resistant cell line, HL-60Res. Mechanistically, in all these cell lines, enzastaurin-ATRA (enz-ATRA) enhanced the protein levels of PU.1, CCAAT/enhancer binding protein $\beta$ (C/EBP $\beta$ ) and C/EBPE. The activity of protein kinase $C \beta$ (PKC $\beta$ ) was suppressed by enz-ATRA treatment in $\mathrm{HL}-60$ and $\mathrm{HL}-60$ Res cells. However, another PKC $\beta$-selective inhibitor mimicked the cellular and molecular effects of enzastaurin only in HL-60 cells. Only in U937 cells, enz-ATRA activated MEK and ERK, and a MEK specific inhibitor suppressed enz-ATRA-triggered differentiation and reduced the protein levels of PU.1, C/EBP $\beta$ and C/EBPE. Enz-ATRA activated Akt in HL-60 and HL60Res cells. However, an Akt inhibitor blocked enz-ATRA-triggered differentiation and restored the protein levels of PU.1, C/EBP $\beta$ and C/EBPE only in HL-60Res cells. Therefore, PKC $\beta$ inhibition, MEK/ERK and Akt activation are involved in enz-ATRA-induced differentiation in HL-60, U937 and HL-60Res cells, respectively by modulation of the protein levels of C/EBP $\beta, C / E B P \varepsilon$ and PU.1.

\section{Conclusions}

Enzastaurin, at the clinically achievable concentration, enhances ATRA-induced differentiation of AML cells by PKC $\beta$ inhibition, MEK/ERK and Akt activation. This study may provide a potential therapeutic strategy for AML patients.

\section{Background}

Acute myeloid leukemia (AML) accounts for $80 \%$ of adult acute leukemia [1]. The median age at diagnosis is about 70 years with 5 -year survival rate of only $10 \%$ for the patients above the age of 60 years while $40 \%$ for younger patients (18-60 years) [1]. Due to the improvement of lifespan of the general population, AML is predicted to increase $38 \%$ in elder patients by 2031 . Over the last four decades, cytarabine/anthracycline-based chemotherapy has been the main therapeutic strategy for AML patients resulting in the remission rate of $60-85 \%$ for patients younger than 60 years of age and $40-60 \%$ for elder patients [1]. However, most patients relapse and become resistant to the treatment. As mentioned above, survival is worse for elder patients who neither can tolerate intensive treatment nor are suitable for stem cell transplantation. Therefore, there is a pressing need to develop new therapeutic strategy for AML patients. Over the last couple of years, some new genetic driver mutations in AML have been identified and several mutation-targeted agents with promising results in clinical trials have been developed [2]. However, only a small portion of AML patients can benefit from these novel treatment strategies. Thus, the development of other effective anti-AML therapies is still required.

Differentiation therapy, which clears tumor bulk by terminal maturation with relatively less severe side effects, may be an alternative to chemotherapy in this circumstance. All-trans retinoic acid (ATRA), the active metabolite of vitamin A, has been successfully applied in the treatment of acute promyelocytic leukemia (APL) by differentiation induction [3]. However, due to the complicated physiopathology of non-APL AML, the clinical trial of ATRA in AML had disappointing results [4]. Since ATRA is a master regulator of myeloid cell differentiation, research strategies to extend the efficacy of ATRA-based therapy to non-APL AML is ongoing. Nucleophosmin1 (NPM1) mutation without FLT3 internal tandem duplications (FLT3-ITD), isocitrate dehydrogenase 1 (IDH1) R132H mutation or over expression of ecotropic viral integration site 1 (EVI-1) have been demonstrated to increase the response of non-APL AML cells to ATRA [5-7], suggesting that AML patients with certain genetic alteration might benefit from ATRA-based therapy. Combination of ATRA with chemotherapy, epigenetic modifiers or arsenic trioxide may be a rational approach to some AML patients [8]. Alternative strategies to increase the expression or the activity of retinoic acid receptor a (RARa) or inhibit its degradation have shown to restore the sensitivity of AML cells to ATRA in vitro or in vivo [8].

MEK/ERK pathway is required for myeloid differentiation induced by certain cytokine and ATRA-triggered differentiation in HL-60 and APL cells [9-12]. Except for limited studies demonstrating that Src inhibitors can promote ATRA-induced differentiation in AML cells by MEK/ERK, the MEK/ERK pathway is rarely used to improve ATRA sensitivity in AML cells $[13,14]$. Since MEK/ERK is an important cytoplasmic pathway for myeloid differentiation, it may serve as a target for enhancing ATRA sensitivity in AML cells. Enzastaurin, a derivative of protein kinase C (PKC) pan-inhibitor 
staurosporine, has been designed to suppress the activation of PKC 3 [15]. It has been proven to be safe and well tolerated in multiple clinical trials, and has shown promising anti-cancer activity [15]. Moreover, it can also reverse ATRA resistance and synergize with ATRA to induce differentiation in ATRAresistant APL cells via MEK/ERK [16]. However, whether enzastaurin can promote ATRA-induced differentiation in AML cells has not yet been investigated.

In this study, non-APL AML cell lines HL-60, U937 and ATRA-resistant HL-60 cell line HL-60Res were used as in vitro models. To be pointed out, these cell lines are all without NPM1 mutation which is associated with increased responsiveness to ATRA [17, 18]. Clinical achievable concentration of enzastaurin enhanced ATRA-induced differentiation in HL-60, U937 and non-APL AML primary cells while it also reversed ATRA resistance in HL-60Res cells. Mechanistically, different pathways were involved in. PKC $\beta$ inhibition, MEK/ERK and AKT regulated the combination of enzastaurin and ATRA (enz-ATRA) induced differentiation in HL-60, U937 and HL-60Res cells, respectively. PU.1, CCAAT/enhancer binding protein $\beta$ (C/EBP $\beta$ ) and C/EBP $\varepsilon$ were the downstream molecules of these signaling pathways.

\section{Methods}

\section{Reagents}

ATRA was obtained from Sigma-Aldrich (St Louis, MO, USA). Enzastaurin, trametinib and Ly294002 were purchased from Selleckchem Chemicals (Houston, TX, USA). A PKC $\beta$ inhibitor was obtained from Merck (Darmstadt, Germany). All reagents were dissolved in dimethyl sulfoxide (DMSO).

\section{Primary cells and cell culture}

Bone marrow samples were collected at the time of diagnosis at the Department of Hematology of Ruijin Hospital. Informed consent was obtained from all patients in accordance with the Declaration of Helsinki, and the study was approved by the Medical Science Ethic committee of Shanghai Jiao Tong University School of Medicine. Mononuclear cells were isolated by density gradient centrifugation using Ficoll-Paque Plus (GE healthcare biosciences, Uppsala, Sweden) and maintained in Iscove's Modified Dulbecco's Medium(IMDM)(GE healthcare bio-sciences) supplemented with 20\% fetal bovine serum (GE healthcare bio-sciences), $10 \mathrm{ng} / \mathrm{mL}$ recombinant human interleukin-3 (rhlL-3), $10 \mathrm{ng} / \mathrm{mL}$ rhlL- 6 and $50 \mathrm{ng} / \mathrm{mL}$ recombinant human stem cell factor (rh SCF) (PeproTech Inc China, Suzhou, Jiangsu, China).

HL-60 and HL-60Res cells were cultured in IMDM, supplemented with 20\% fetal bovine serum while U937 cells were cultured in RPMI-1640 medium (GE healthcare bio-sciences), supplemented with $10 \%$ fetal bovine serum in a humidified atmosphere of $95 \%$ air and $5 \% \mathrm{CO} 2$ at $37{ }^{\circ} \mathrm{C}$.

\section{Annexin- $\mathrm{V}$ analysis}

According to instructions provided in the Annexin V-7AAD Apoptosis Detection Kit (BD Biosciences Pharmingen, San Diego, CA, USA), $5 \times 10^{5}$ cells were harvested and washed with binding buffer. Subsequently, cells were incubated with $5 \mu \mathrm{L}$ 7-Amino-Actinomycin and $5 \mu \mathrm{L}$ annexin-V in the dark at room temperature for $15 \mathrm{~min}$. Fluorescent intensities were evaluated by flow cytometry (EPICS XL, Coulter, Hialeah, FL, USA).

\section{Cell differentiation assays}

Cell maturation was determined by cellular morphology and the content of cell surface differentiation-related antigen CD11b. Morphology was evaluated with May-Grunwald-Giemsa's staining and observed at 1000x magnification. The expression of cell surface differentiation-related antigen CD11b (Coulter, Marseilles, France) was determined by flow cytometry (EPICS XL).

\section{Western-blotting analysis}

After lysed with RIPA buffer (Sigma-Aldrich) and centrifuged at 13,000 rpm for 10 min at $4{ }^{\circ} \mathrm{C}$, supernants were collected and quantified by Bio-Rad Dc protein assay (Bio-Rad Laboratories, Hercules, CA, USA). 20 or $50 \mu$ g protein extracts were loaded onto $8 \%$ SDS-polyacrylamide gel, subjected to electrophoresis, and transferred to polyvinylidene difluoride membranes (GE Healthcare UK Ltd, Buckinghamshire, UK). Blocking with $5 \%$ nonfat milk or BSA in PBS, the membranes were incubated with the following primary antibodies: C/EBP, C/EBPE, PU. 1 from Santa Cruz Biotech (Santa Cruz, CA, USA); phospho-p44/42 Erk1/2 (Thr202/Try204), phospho-MEK1/2 (Ser218/ 222), Phospho-PKC (pan) ( $\beta$ II Ser660), Phospho-PKCa/ $\beta$ II (Thr638/641), Phospho-Akt (Ser473), Phospho-Akt (Thr308) from Cell Signaling Technology (Beverly, MA, USA); GAPDH from Proteintech (Rosemont, IL, USA). Then membranes were probed with horseradish peroxidase (HRP)-conjugated secondary antibody (GE Healthcare UK Ltd). Immunocomplexes were visualized with chemiluminescence kit (GE Healthcare UK Ltd). To detect Erk1/2, MEK1/2, PKC $\beta$ and AKT, the same membrane incubated with accordingly phosphorylated antibody was stripped with stripping buffer ( $2 \%$ SDS, $100 \mathrm{mM}$ beta-mercaptoethanol, $50 \mathrm{mM}$ Tris, pH6.8), followed by blocking and probing respectively with anti-Erk1/2 (Cell Signaling Technology), anti-MEK1/2 (Cell Signaling Technology), anti-PKC $\beta$ (Santa Cruz Biotech) or anti-AKT (Cell Signaling Technology).

\section{Statistical analysis}

For cell growth, cell survival, Annexin-V assay and histogram of CD11b, values are expressed as mean \pm SD, $p$ values are mentioned in the corresponding figure legends. Chi-square test $(n=20,000)$ was used to analyze the flow-cytometric analysis of $C D 11 b, P<0.05$ was taken to indicate statistical significance. 


\section{Results}

Enzastaurin enhances ATRA-induced differentiation in HL-60,U937 cells and patient-derived AML blasts while it also reverses the ATRA resistance in HL-60Res cells.

Since $2 \mu \mathrm{M}$ has been demonstrated to be the clinical achievable concentration of enzastaurin [19], such concentration was used as the maximum concentration of enzastaurin in all the cell lines. Only $2 \mu \mathrm{M}$ enzastaurin alone or in combination with ATRA inhibited cell growth in HL-60 and U937 cells (Additional file1: Figure S1a and c) while the proliferation was not affected with any treatment in HL-60Res cells (Additional file1: Figure S1b). The cell viability was maintained above $95 \%$ with any treatment in all the cell lines (Additional file1: Figure S1d-f). Meanwhile, the content of Annexin $\mathrm{V}^{+}$cells only increased slightly with some treatments in all the cell lines (Additional file1: Figure S1g-i).

Morphologically, as illustrated in Fig. 1a, all the cell lines presented a characteristic morphology of primitive cells such as round nucleus and large nuclear/cytoplasm ratio. With ATRA treatment for 3 days in HL-60 and U937 cells, some cells displayed decreased nuclear/cytoplasm ratio with kidneyshape nuclei. However, there was no obvious change in HL-60Res cells with ATRA treatment for 10 days. More matured cells were presented in all the cell lines with the combination of any concentration of enzastaurin and ATRA, especially with co-treatment of ATRA and $2 \mu M$ enzastaurin (Fig. $1 a$ ). Consistent with the morphology, a synergistic effect of enzastaurin and ATRA on the content of CD11 $\mathrm{b}^{+}$cells was also observed in a dose-dependent manner in all the cell lines (Fig. 1b-e). Therefore, enzastaurin enhanced ATRA-induced differentiation of HL-60 and U937 cells while restored ATRA sensitivity in HL-60Res cells. For non-APL AML primary cells, 5 out of 9 samples were AML-M4 and AML-M5 (Table 1). In 4 out of these 5 samples, enzastaurin enhanced ATRA-induced differentiation as assayed by morphology and the content of CD11 ${ }^{+}$cells (Fig. $2 a$ and $b$ ). In samples diagnosed as AML-M1 (No.2), AML-M2 (No.8 and No.9), MDS transforming to AML (No.7) and AML-M5 with AML-ETO fusion gene and c-kit mutation (No.6), such effect was not observed (Table 1). One difference between these two groups was that 4 cases that were effective to enz-ATRA had partial response to ATRA, while 5 cases that were invalid to enz-ATRA had no response to ATRA (Table 1). Thus, enzastaurin enhanced ATRA-induced differentiation in some AML primary cells.

Table 1

Patients data and response to enzastaurin and/or ATRA

\begin{tabular}{|c|c|c|c|c|c|c|c|c|c|c|c|}
\hline \multirow[t]{2}{*}{ No. } & \multirow[t]{2}{*}{$\operatorname{sex}$} & \multirow[t]{2}{*}{ age } & \multirow[t]{2}{*}{ karyotype } & \multirow[t]{2}{*}{ Gene mutation/fusion } & \multirow[t]{2}{*}{ Blast(\%) } & \multirow[t]{2}{*}{ WBC(x109/L) } & \multirow{2}{*}{$\begin{array}{l}\text { FAB } \\
\text { classification }\end{array}$} & \multicolumn{4}{|c|}{ CD11b + cells(\%) } \\
\hline & & & & & & & & DMSO & RA & EN & $\begin{array}{l}\text { EN } \\
+ \\
\text { RA }\end{array}$ \\
\hline 1 & M & 65 & $\begin{array}{l}46, X Y, t(6 ; 11) \\
\text { (q27;q23) }\end{array}$ & MLL-AF6 & 85.5 & 9.17 & AML-M4 & 9.53 & 33.4 & 33.9 & 56.7 \\
\hline 2 & M & 55 & $46, X Y$ & $\begin{array}{l}\text { C/EBPa G141C mutation, } \\
\text { C/EBPa P192_H193 inserts } \\
\text { PP, C/EBPa A303_K313 } \\
\text { duplication, C/EBPa } \\
\text { Q305_R306 inserts } \\
\text { HNVETQQKAKQ }\end{array}$ & 88 & 288 & AML-M1 & 0.6 & 3.8 & 0.2 & 5.5 \\
\hline 3 & M & 57 & $46, X Y$ & $\begin{array}{l}\text { FLT3Y599_D600 inserts } \\
\text { GSTGSSDNEYFYVDFREYEY }\end{array}$ & 50 & 97.73 & AML-M5 & 19.4 & 20.8 & 26.9 & 37.5 \\
\hline 4 & $\mathrm{~F}$ & 44 & $\begin{array}{l}48 \sim \\
49, X X, t(10 ; 11) \\
\text { (p12;q23),+21*3 }\end{array}$ & MLL-AF10, N-RAS mutation & 83 & 7.8 & AML-M5a & 3.33 & 24 & 9.87 & 33.4 \\
\hline 5 & M & 76 & $\begin{array}{l}45 \sim \\
47, \mathrm{XY},-7,+\mathrm{M} 1 \sim \\
\mathrm{M} 6\end{array}$ & NRASG12S mutation & 73 & 74.71 & AML-M4b & 10.4 & 53 & 20.4 & 63.5 \\
\hline 6 & $\mathrm{~F}$ & 55 & $\begin{array}{l}45, X,- \\
X, t(8 ; 21 ; 12) \\
\text { (q22;q22p13) }\end{array}$ & $\begin{array}{l}\text { AML1-ETO,C-KIT T380 } \\
\text { duplication }\end{array}$ & 86 & 14 & AML-M5 & 3.2 & 3.9 & 9.3 & 9 \\
\hline 7 & M & 51 & $46, X Y$ & $\begin{array}{l}D N M T 3 A \mathrm{~N} \text {-terminal } \\
\text { catalytic domain mutation }\end{array}$ & ND & 3.79 & $\begin{array}{l}\text { MDS } \\
\text { transforming } \\
\text { to AML }\end{array}$ & 3 & 3.8 & 4.9 & 5.8 \\
\hline 8 & $\mathrm{~F}$ & 56 & $46, X X$ & C/EBPa K313 duplication & 54 & 8.45 & AML-M2a & 2.2 & 1.9 & 7.2 & 8 \\
\hline 9 & $\mathrm{~F}$ & 38 & $46, X X$ & C/EBPa Q305P mutation & 36.5 & 6.12 & AML-M2 & 0.8 & 0.8 & 1.8 & 2.6 \\
\hline
\end{tabular}

Enzastaurin enhances ATRA-induced differentiation in HL-60 cells by inhibition of PKC $\beta$. 
To investigate the mechanisms of enz-ATRA treatment-triggered differentiation in these three cell lines, we used $2 \mu \mathrm{M}$ enzastaurin in the following studies. Since enzastaurin has been designed to suppress the activation of PKC $\beta$ [15], we first studied the role of PKC $\beta$ in enz-ATRA-induced differentiation. Phosphorylation of Ser660 or Thr641 is essential for activation of PKC $\beta$ [20]. As shown in Fig. 3a, comparing with ATRA treatment, with enz-ATRA treatment, the phosphorylation of PKC 3660 was decreased in HL-60 cells while the phosphorylation of PKC $\beta$ T641 was reduced in HL60Res cells. However, in U937 cells, neither the phosphorylation of PKC 3660 nor that of PKC $\beta$ T641 was reduced with enz-ATRA treatment comparing with ATRA treatment. Thus, enzastaurin inhibited PKC $\beta$ in $\mathrm{HL}-60$ and $\mathrm{HL}-60$ Res cells. To confirm the role of PKC $\beta$, another PKC $\beta$ inhibitor was combined with ATRA to examine whether it could mimic the effect of enzastaurin to augment ATRA-induced differentiation. $500 \mathrm{nM}, 200 \mathrm{nM}$ and $100 \mathrm{nM}$ PKC $\beta$ inhibitor was used in HL-60, HL-60Res and U937 cells, respectively, with no obvious effects on survival. To note, such concentration of PKC $\beta$ is $5-25$ fold higher than the $\mathrm{IC}_{50}$ to inhibit $\mathrm{PKC} \beta \mathrm{I}$ and $\mathrm{PKC} \beta \mathrm{II}$ as indicated in the instructions. Like enz-ATRA treatment, fully differentiated cells with lobed nuclei accompanied by markedly decreased nuclear/cytoplasm ratio were presented in HL-60 cells with PKC $\beta$ inhibitor-ATRA treatment for 4 days (Fig. 3b). Moreover, comparing with enz-ATRA treatment, the content of CD11 b cells was increased to the similar level in HL-60 cells with PKC $\beta$ inhibitor-ATRA treatment (Fig. $3 c$ and d). Similar to enz-ATRA treatment, comparing with ATRA treatment, the phosphorylation of PKC $\beta$ S660 was reduced with PKC $\beta$ inhibitor in $\mathrm{HL}-60$ cells (Fig. 3e). Thus, by suppression of $\mathrm{PKC} \beta, \mathrm{PKC} \beta$ inhibitor could enhance ATRA-triggered differentiation in HL-60 cells just like enzastaurin. These results suggested that PKC $\beta$ inhibition might regulate enzastaurin-enhanced ATRA-triggered differentiation in HL-60 cells. However, PKC $\beta$ inhibitor could neither elevate ATRA-triggered differentiation in U937 cells nor restore ATRA sensitivity in HL-60Res cells as evaluated by morphology (Fig. 3b) and the content of CD11 $b^{+}$cells (Fig. 3c and d). Therefore, PKC $\beta$ may not be involved in enz-ATRA treatment-triggered differentiation in U937 and HL-60Res cells.

\section{PKC $\beta$ inhibition and MEK/ERK activation are involved in enz-ATRA-induced differentiation in HL-60 and U937 cells, respectively by upregulation of the protein levels of C/EBP $\beta, C / E B P \varepsilon$ and PU.1.}

To further survey the mechanisms of enz-ATRA treatment-triggered differentiation, we studied several proteins and signal pathways involved in ATRAinduced differentiation in HL-60 cells or granulocytes. As mentioned above, MEK/ERK signal pathway regulates certain cytokine-induced myeloid differentiation and ATRA-triggered granulocytic differentiation in APL cells and HL-60 cells [9-12]. C/EBP $3, \mathrm{C} / \mathrm{EBP} \varepsilon$ and PU.1 are required for the maturation of the myeloid lineages, as well as ATRA-induced differentiation in APL cells [21-24]. Moreover, by MEK/ERK modulating the protein levels of C/EBP $\beta, C / E B P \varepsilon$ and PU.1, some medicines including enzastaurin synergize with ATRA to induce differentiation in ATRA-resistant APL cells [16, 2527]. As Fig. 4 shown, comparing with ATRA treatment, the protein levels of C/EBP $\beta, C / E B P \varepsilon$ and PU. 1 were increased remarkably by enz-ATRA treatment in all the cell lines. Meanwhile, comparing with ATRA treatment, PKC $\beta$ inhibitor-ATRA treatment also augmented the protein levels of $C / E B P \beta$, C/EBP $\varepsilon$ and PU. 1 in HL-60 cells. It was suggested that enzastaurin promoted ATRA up-regulated protein levels of C/EBP $\beta$, C/EBP $\varepsilon$ and PU. 1 by PKCinhibition to enhance ATRA induced-differentiation in HL-60 cells. However, comparing with ATRA, the phosphorylation levels of MEK and ERK were enhanced only in $U 937$ cells with enz-ATRA treatment. In HL-60 and HL-60Res cells, ATRA phosphorlated MEK and ERK, while enz-ATRA did not elevate their phosphorylation levels. Trametinib, a highly specific and potent MEK1/2 inhibitor [28] did attenuate MEK activity in all the cell lines, as determined by Western-blotting of phosphorylated ERK1/2 (Fig. 5a). With trametinib pretreatment, fully differentiated cells with lobed nuclei and a decreased nuclear/cytoplasm ratio were replaced by primitive cells with round nuclei and a large nuclear/cytoplasm ratio in U937 cells (Fig. $5 \mathrm{~b}$ ). The content of CD $11 b^{+}$cells was also significant suppressed by trametinib in U937 cells (Fig. $5 \mathrm{c}$ and d). Moreover, in the presence of trametinib, enz-ATRA treatmentenhanced protein levels of C/EBP $\beta, C / E B P \varepsilon$ and PU.1 were remarkably decreased in U937 cells (Fig. 5e). Thus, enz-ATRA treatment-induced differentiation in U937 cells via MEK/ERK modulation of the protein levels of C/EBP $\beta, C / E B P \varepsilon$ and PU.1. Trametinib slightly inhibited enz-ATRA treatment-triggered differentiation in HL-60 cells while unexpectedly augmented enz-ATRA treatment-induced differentiation in HL-60Res cells as evaluated by morphology (Fig. 5b) and the content of CD11 b ${ }^{+}$cells (Fig. 5c and d). Therefore, MEK/ERK signal pathway may not regulate enz-ATRA treatment-triggered differentiation in HL-60 and HL-60Res cells.

\section{Akt activation positively regulates enz-ATRA-induced differentiation in HL-60Res cells by modulation of the protein levels of C/EBP $\beta$, C/EBP $\varepsilon$ and PU.1.}

Besides MEK/ERK, PI3K/AKT is another signal pathway demonstrated to be essential for ATRA-induced differentiation in HL-60 cells [29]. Phosphorylation of Ser473 or Thr308 is essential for activation of Akt [30]. In HL-60 cells, ATRA treatment for $48 \mathrm{~h}$ phosphorylated Akt at Ser473 and Thr308 while enz-ATRA treatment enhanced ATRA-promoted phosphorylation of both sites (Fig. 6a). In HL-60Res cells, ATRA did not enhance the phosphorylation of Akt, but enz-ATRA treatment for $72 \mathrm{~h}$ increased the phosphorylation of Akt at Ser473 (Fig. 6a). LY294002, the inhibitor of PI3K, did attenuate the activation of Akt in both cell lines (Fig. 6b). However, it suppressed enz-ATRA-induced differentiation in HL-60Res cells but not in HL-60 cells as determined by morphology (Fig. 6c) and the content of CD11 b+ cells (Fig. 6d and e). Moreover, with LY294002 pretreatment, the protein levels of C/EBP $\beta, C / E B P \varepsilon$ and PU.1 enhanced by enz-ATRA in HL-60Res cells were significantly reduced (Fig. 6f). Therefore, AKT was not involved in enzATRA treatment-triggered differentiation in HL-60 cells. However, enz-ATRA treatment induced differentiation in HL-60Res cells via Akt modulation of the protein levels of C/EBPß, C/EBPE and PU.1.

\section{Discussion}

In this study, we demonstrated that enzastaurin enhanced ATRA-induced differentiation in HL-60, U937 and non-APL AML primary cells as well as reversed ATRA-resistance in HL-60Res cells. As mentioned above, these three cell lines are all without NPM1 mutation, the marker of ATRA sensitivity $[17,18]$. As the ATRA resistant cells, HL-60Res cells may better reflect the clinical effect of ATRA and enz-ATRA in non-APL AML patients. Moreover, the concentration of enzastaurin used in this study is clinical achievable [19]. Taken together, it indicates that such combination may provide a potential

Page 5/14 
therapy strategy for some AML patients. For patient-derived AML blasts, in 4 out of 5 AML-M4 and AML-M5 samples, enzastaurin enhanced ATRAinduced differentiation. It seemed that such combination might be effective in AML-M4 and AML-M5 patients. However, we also observed that the effectiveness of enz-ATRA in AML primary cells might be associated with ATRA sensitivity. Therefore, the association of sensitivity to enz-ATRA treatment with AML subtype or ATRA sensitivity needs to be further surveyed in a large number of patient samples. Due to the limited number of patient samples, the association of sensitivity to enz-ATRA treatment with age, gender, chromosomal and genetic changes has not been investigated.

PKC is a family of serine/threonine kinases, consisting of 13 isozymes that play a crucial role in regulation of proliferation, differentiation, apoptosis, cell migration and gene expression. The role of different PKC isozymes in granulocytic differentiation is quite controversial. For ATRA-induced granulocytic differentiation in $\mathrm{HL}-60$ cells, PKCa and PKCßII are activated and positively regulate granulocytic differentiation [31]. However, Zauli G et al reported that only PKCa and PKC 3 but neither PKC $3 I$ nor PKC $\beta$ I showed significant modification upon ATRA treatment in HL-60 cells [32].

Kambhampati $S$ et al showed that only PKC $\delta$ was activated with ATRA treatment in HL-60 cells [33]. For myeloid differentiation, PKCa is suggested not to be required for granulocytic differentiation because its mRNA and protein are decreased with ATRA treatment in HL-60 and undetectable in peripheral blood neutrophils [34]. However, PLCY2 and PKC are demonstrated to be crucial upstream signals that modulate myelopoiesis by G-CSF [35]. In this study, the inhibition of $\mathrm{PKC} \beta$ by either enzastaurin or another PKC $\beta$ inhibitor did enhance ATRA-induced differentiation in $\mathrm{HL}-60$ cells. It indicates that PKC $\beta$ may negatively regulate ATRA-induced differentiation in $\mathrm{HL}-60$ cells. Further study showed that enzastaurin and PKC $\beta$ inhibitor both increased ATRA-enhanced protein levels of C/EBP, $\mathrm{C} / \mathrm{EBP} \varepsilon$ and PU.1 in HL-60 cells. It suggests that PKC $\beta$ inhibition may control enzastaurin-enhanced differentiation in HL-60 cells by modulation of the protein levels of C/EBP,$C / E B P \varepsilon$ and PU.1. PKC positively or negatively regulates the transcriptional activity of $C / E B P \beta$ by phosphorylation on different site $[36,37]$. In addition, $C / E B P \beta$ can trigger the expressions of C/EBP $\varepsilon$ and PU.1 in ATRA-triggered differentiation in APL cells $[22,23]$. PU.1 can also directly activate the transcription of C/EBPE [38]. Therefore, there might be PKC $\beta$ inhibition-C/EBP $\beta$ PU.1-C/EBP $\varepsilon$ cascade in enz-ATRA-induced differentiation in HL-60 cells. PKC $\beta$ was inhibited by enz-ATRA in HL-60Res cells whereas it was not suppressed in $U 937$ cells. Moreover, another PKC $\beta$ inhibitor could not mimic the effect of enzastaurin to enhance ATRA-induced differentiation in both cell lines. Thus, PKC $\beta$ might not be involved in enz-ATRA-induced differentiation in these two cell lines. In fact, PKC $\beta$-independent effect of enzastaurin is not rare $[16,39-41]$.

Comparing with ATRA, the phosphorylation levels of MEK and ERK were enhanced with enz-ATRA treatment only in U937 cells. Moreover, the specific inhibitor of MEK suppressed enz-ATRA-induced differentiation and the protein levels of C/EBP $\beta, C / E B P \varepsilon$ and PU.1 only in U937 cells. Therefore, MEK/ERK-modulated the protein levels of C/EBP $\beta, C / E B P \varepsilon$ and PU.1 was involved in enz-ATRA-induced differentiation only in U937 cells, but not HL-60 cells or HL-60Res cells. Consistent with this study, staurosporine, the parent compound of enzasataurin, enhances ATRA-induced differentiation in U937 cells also via MEK/ERK-mediated modulation of C/EBPs [42]. MEK and ERK have been demonstrated to promote the expression of C/EBP $\beta$ and regulate the activity of C/EBP $\beta$ and PU.1 [43-45]. Since there is interaction of C/EBP $\beta, C / E B P \varepsilon$ and PU.1 as mentioned above [22, 23, 38], there might be MEK-ERK-C/EBPß-PU.1-C/EBPE cascade in enz-ATRA-induced differentiation in U937 cells.

Akt can positively or negatively control differentiation of leukemia cells depending on cell type and differentiation inducer [31, 46]. In HL-60 cells, Akt activation is required for ATRA-induced differentiation [29]. In the present study, though Akt was activated by enz-ATRA treatment, the inhibition of Akt did not suppress enz-ATRA-triggered differentiation in HL-60 cells. Thus, the involvement of Akt in enz-ATRA-triggered differentiation in HL-60 cells was excluded. In HL-60Res cells, Akt was activated by enz-ATRA treatment and Akt inhibitor suppressed differentiation and the protein levels of C/EBP $\beta$, $\mathrm{C} / \mathrm{EBP} \varepsilon$ and PU.1. Therefore, Akt activation was involved in enz-ATRA-induced differentiation in HL-60Res cells by modulation of the protein levels of $\mathrm{C} / \mathrm{EBP} \beta, \mathrm{C} / \mathrm{EBP} \varepsilon$ and PU.1. Akt induces transcriptional activity and expression of C/EBP $\beta$ and PU.1 [47-50]. In addition, C/EBP $\beta$ can trigger the expressions of C/EBPE and PU.1 [22, 23]. Collectively, there might be Akt-C/EBPB-PU.1-C/EBP $\varepsilon$ cascade in enz-ATRA-induced differentiation in HL60Res cells.

\section{Conclusions}

A clinically achievable concentration of enzastaurin enhances ATRA-induced differentiation of HL-60, U937 and non-APL AML primary cells as well as reverses ATRA-resistance in HL-60Res cells. PKC $\beta$ inhibition, MEK/ERK and Akt activation are involved in enz-ATRA-induced differentiation of HL-60, U937 and HL-60Res cells, respectively by modulating the protein levels of C/EBP $\beta, C / E B P \varepsilon$ and PU.1. This study may provide a potential therapeutic strategy for some AML patients.

\section{Abbreviations}

AML

acute myeloid leukemia; APL:acute promyelocytic leukemia; ATRA:all-trans retinoic acid; C/EBPß:CCAAT/enhancer binding protein $\beta$; DMSO:dimethyl sulfoxide; IDH1:isocitrate dehydrogenase 1; EVI-1:ecotropic viral integration site 1; FLT3-ITD:FLT3 internal tandem duplications; NPM1:Nucleophosmin1; PKC:protein kinase C; RARa:retinoic acid receptor a; rhIL-3:recombinant human interleukin-3; rh SCF:recombinant human stem cell factor

\section{Declarations}

\section{Ethics approval and consent to participate}


This study involved bone marrow samples from AML patients. Informed consent was obtained from all patients in accordance with the Declaration of Helsinki, and the study was approved by the Medical Science Ethic committee of Shanghai Jiao Tong University School of Medicine.

\section{Consent for publication}

Not applicable.

\section{Availabilty of data and materials}

All data generated or analyzed during this study are included in this published

article [and its additional information files].

\section{Competing interests}

The authors declare that they have no competing interests.

\section{Funding}

This work is supported by the Natural Science Foundation of Shanghai (17ZR1417100).

\section{Author contributions}

ZY L, L C, M D, XQ W, Y S, J W and H L carried out the experiments. ZY L prepared all the figures. X C designed the study and wrote the manuscript. All authors read and approved the final manuscript.

\section{Acknowledgements}

Not applicable.

\section{References}

1. Döhner H, Weisdorf DJ, Bloomfield CD. Acute Myeloid Leukemia. N Engl J Med. 2015;373(12):1136-1152.

2. Bohl SR, Bullinger L, Rücker FG. New Targeted Agents in Acute Myeloid Leukemia: New Hope on the Rise. Int J Mol Sci. 2019;20(8):1983.

3. Huang ME, Ye YC, Chen SR, et al. Use of all-trans retinoic acid in the treatment of acute promyelocytic leukemia. Blood. 1988;72(2):567-572.

4. Tallman MS. Differentiating therapy with all-trans retinoic acid in acute myeloid leukemia. Leukemia. 1996;10 Suppl 1:S12-15.

5. Schlenk RF, Döhner K, Kneba M, et al. Gene mutations and response to treatment with all-trans retinoic acid in elderly patients with acute myeloid leukemia. Results from the AMLSG Trial AML HD98B. Haematologica. 2009(1);94:54-60.

6. Boutzen $\mathrm{H}$, Saland $\mathrm{E}$, Larrue $\mathrm{C}$, et al. Isocitrate dehydrogenase 1 mutations prime the all-trans retinoic acid myeloid differentiation pathway in acute myeloid leukemia. J Exp Med. 2016;213(4):483-497.

7. Verhagen HJ, Smit MA, Rutten A, et al. Primary acute myeloid leukemia cells with overexpression of EVI-1 are sensitive to all-trans retinoic acid. Blood. 2016;127(4):458-463.

8. Ni X, Hu G, Cai X. The success and the challenge of all-trans retinoic acid in the treatment of cancer. Crit Rev Food Sci Nutr. 2019;59(sup1):S71-s80.

9. Miranda MB, McGuire TF, Johnson DE. Importance of MEK-1/-2 signaling in monocytic and granulocytic differentiation of myeloid cell lines. Leukemia. 2002;16:683-692.

10. Miranda MB, Xu H, Torchia JA, Johnson DE. Cytokine-induced myeloid differentiation is dependent on activation of the MEK/ERK pathway. Leuk Res. 2005;29:1293-1306.

11. Weng XQ, Sheng Y, Ge DZ, Wu J, Shi L, Cai X. RAF-1/MEK/ERK pathway regulates ATRA-induced differentiation in acute promyelocytic leukemia cells through C/EBPß, C/EBPE and PU.1. Leuk Res. 2016;45:68-74.

12. Yen A, Roberson MS, Varvayanis S, Lee AT. Retinoic acid induced mitogen-activated protein (MAP)/extracellular signal-regulated kinase (ERK) kinase-dependent MAP kinase activation needed to elicit HL-60 cell differentiation and growth arrest. Cancer Res. 1998;58(14):3163-3172.

13. Congleton J, MacDonald R, Yen A. Src inhibitors, PP2 and dasatinib, increase retinoic acid-induced association of Lyn and c-Raf (S259) and enhance MAPK-dependent differentiation of myeloid leukemia cells. Leukemia. 2012;26(6):1180-1188.

14. Kropf PL, Wang L, Zang Y, Redner RL, Johnson DE. Dasatinib promotes ATRA-induced differentiation of AML cells. Leukemia. 2010;24(3):663-665.

15. Bourhill T, Narendran A, Johnston RN. Enzastaurin: A lesson in drug development. Crit Rev Oncol Hematol. 2017;112:72-79.

16. Liang C, Ding M, Weng XQ, et al. Combination of enzastaurin and ATRA exerts dose-dependent dual effects on ATRA-resistant acute promyelocytic leukemia cells. Am J Cancer Res. 2019;9(5):906-926.

17. Bunaciu RP, MacDonald RJ, Gao F, et al. Potential for subsets of wt-NPM1 primary AML blasts to respond to retinoic acid treatment. Oncotarget. 2017;9(3):4134-4149. 
18. Pollock SL, Rush EA, Redner RL. NPM-RAR, not the RAR-NPM reciprocal t(5;17)(q35;q21) acute promyelocytic leukemia fusion protein, inhibits myeloid differentiation. Leuk Lymphoma. 2014;55(6):1383-1387.

19. KreisI TN, Kim L, Moore K, et al. A phase I trial of enzastaurin in patients with recurrent gliomas. Clin Cancer Res. 2009;15:3617-3623.

20. Keranen LM, Dutil EM, Newton AC. Protein kinase C is regulated in vivo by three functionally distinct phosphorylations. Curr Biol. 1995;5:13941403.

21. Lekstrom-Himes JA. The role of C/EBP(epsilon) in the terminal stages of granulocyte differentiation. Stem Cells. 2001;19:125-133.

22. Duprez E, Wagner K, Koch H, Tenen DG. C/EBPbeta: a major PML-RARA-responsive gene in retinoic acid-induced differentiation of APL cells. Embo j. 2003;22:5806-5816.

23. Mueller BU, Pabst T, Fos J, et al. ATRA resolves the differentiation block in $\mathrm{t}(15 ; 17)$ acute myeloid leukemia by restoring PU.1 expression. Blood. 2006;107:3330-3338.

24. Park DJ, Chumakov AM, Vuong PT, et al. CCAAT/enhancer binding protein epsilon is a potential retinoid target gene in acute promyelocytic leukemia treatment. J Clin Invest. 1999;103:1399-1408.

25. Liang C, Ding M, Weng XQ, Sheng Y, Wu J, Cai X. The combination of UCN-01 and ATRA triggers differentiation in ATRA resistant acute promyelocytic leukemia cell lines via RAF-1 independent activation of MEK/ERK. Food Chem Toxicol. 2019;126:303-312.

26. Ding $M$, Weng XQ, Sheng Y, Wu J, Liang C, Cai X. Dasatinib synergizes with ATRA to trigger granulocytic differentiation in ATRA resistant acute promyelocytic leukemia cell lines via Lyn inhibition-mediated activation of RAF-1/MEK/ERK. Food Chem Toxicol. 2018;119:464-478.

27. Ge DZ, Sheng Y, Cai X. Combined staurosporine and retinoic acid induces differentiation in retinoic acid resistant acute promyelocytic leukemia cell lines. Sci Rep. 2014;4:4821.

28. Yamaguchi T, Kakefuda R, Tajima N, Sowa Y, Sakai T. Antitumor activities of JTP-74057 (GSK1120212), a novel MEK1/2 inhibitor, on colorectal cancer cell lines in vitro and in vivo. Int J Oncol. 2011;39(1):23-31.

29. Bertagnolo V, Neri LM, Marchisio M, Mischiati C, Capitani S. Phosphoinositide 3-kinase activity is essential for all-trans-retinoic acid-induced granulocytic differentiation of HL-60 cells. Cancer Res. 1999;59(3):542-546.

30. Brenner AK, Andersson Tvedt TH, Bruserud Ø. The Complexity of Targeting PI3K-Akt-mTOR Signalling in Human Acute Myeloid Leukaemia: The Importance of Leukemic Cell Heterogeneity, Neighbouring Mesenchymal Stem Cells and Immunocompetent Cells. Molecules. 2016;21(11):1512.

31. Yamada O, Ozaki K, Nakatake M, et al. Akt and PKC are involved not only in upregulation of telomerase activity but also in cell differentiationrelated function via mTORC2 in leukemia cells. Histochem Cell Biol. 2010;134(6):555-563.

32. Zauli G, Visani G, Bassini A, et al. Nuclear translocation of protein kinase C-alpha and -zeta isoforms in HL-60 cells induced to differentiate along the granulocytic lineage by all-trans retinoic acid. Br J Haematol. 1996;93(3):542-550.

33. Kambhampati S, Li Y, Verma A, et al. Activation of protein kinase C delta by all-trans-retinoic acid. J Biol Chem. 2003;278(35):32544-32551.

34. Devalia V, Thomas NS, Roberts PJ, Jones HM, Linch DC. Down-regulation of human protein kinase $\mathrm{C}$ alpha is associated with terminal neutrophil differentiation. Blood. 1992;80(1):68-76.

35. Barbosa CM, Bincoletto C, Barros CC, Ferreira AT, Paredes-Gamero EJ. PLCY2 and PKC are important to myeloid lineage commitment triggered by M-SCF and G-CSF. J Cell Biochem. 2014;115(1):42-51.

36. Trautwein C, Caelles C, van der Geer P, Hunter T, Karin M, Chojkier M. Transactivation by NF-IL6/LAP is enhanced by phosphorylation of its activation domain. Nature. 1993;364(6437):544-547.

37. Trautwein C, van der Geer P, Karin M, Hunter T, Chojkier M. Protein kinase A and C site-specific phosphorylations of LAP (NF-IL6) modulate its binding affinity to DNA recognition elements. J Clin Invest. 1994;93(6):2554-2561.

38. Yoshida H, Ichikawa H, Tagata Y, et al. PML-retinoic acid receptor alpha inhibits PML IV enhancement of PU.1-induced C/EBPepsilon expression in myeloid differentiation. Mol Cell Biol. 2007;27:5819-5834.

39. Querfeld C, Rizvi MA, Kuzel TM, et al. The selective protein kinase C beta inhibitor enzastaurin induces apoptosis in cutaneous T-cell lymphoma cell lines through the AKT pathway. J Invest Dermatol. 2006;126:1641-1647.

40. Rizvi MA, Ghias K, Davies KM, et al. Enzastaurin (LY317615), a protein kinase Cbeta inhibitor, inhibits the AKT pathway and induces apoptosis in multiple myeloma cell lines. Mol Cancer Ther. 2006;5:1783-1789.

41. Ruvolo PP, Zhou L, Watt JC, et al. Targeting PKC-mediated signal transduction pathways using enzastaurin to promote apoptosis in acute myeloid leukemia-derived cell lines and blast cells. J Cell Biochem. 2011;112:1696-1707.

42. Shi L, Weng XQ, Sheng Y, Wu J, Ding M, Cai X. Staurosporine enhances ATRA-induced granulocytic differentiation in human leukemia U937 cells via the MEK/ERK signaling pathway. Oncol Rep. 2016;36ه5:3072-3080.

43. Lee S, Shuman JD, Guszczynski T, et al. RSK-mediated phosphorylation in the C/EBP\{beta\} leucine zipper regulates DNA binding, dimerization, and growth arrest activity. Mol Cell Biol. 2010;30:2621-2635.

44. Lu J, Wu DM, Zheng YL, et al. Troxerutin counteracts domoic acid-induced memory deficits in mice by inhibiting CCAAT/enhancer binding protein $\beta$-mediated inflammatory response and oxidative stress. J Immunol. 2013;190:3466-3479.

45. Murakami M, Ito H, Hagiwara K, et al. Sphingosine kinase 1/S1P pathway involvement in the GDNF-induced GAP43 transcription. J Cell Biochem. 2011;112:3449-3458. 
46. Huang YM, Zheng Y, Li JG, et al. Lentivirus-mediated RNA interference targeting FAMLF-1 inhibits cell growth and enhances cell differentiation of acute myeloid leukemia partially differentiated cells via inhibition of AKT and c-MYC. Oncotarget. 2017;8(60):101372-101382.

47. Breig O, Théoleyre O, Douablin A, Baklouti F. Subtle distinct regulations of late erythroid molecular events by PI3K/AKT-mediated activation of Spi1/PU.1 oncogene autoregulation loop. Oncogene. 2010;29(19):2807-2816.

48. Rieske P, Pongubala JM. AKT induces transcriptional activity of PU.1 through phosphorylation-mediated modifications within its transactivation domain. J Biol Chem. 2001;276(11):8460-8468.

49. Guo Y, He X, Zhang M, et al. Reciprocal control of ADAM17/EGFR/Akt signaling and miR-145 drives GBM invasiveness. J Neurooncol. 2020;147(2):327-337.

50. Wu DW, Wu TC, Chen CY, Lee H. PAK1 Is a Novel Therapeutic Target in Tyrosine Kinase Inhibitor-Resistant Lung Adenocarcinoma Activated by the PI3K/AKT Signaling Regardless of EGFR Mutation. Clin Cancer Res. 2016;22(21):5370-5382.

\section{Figures}

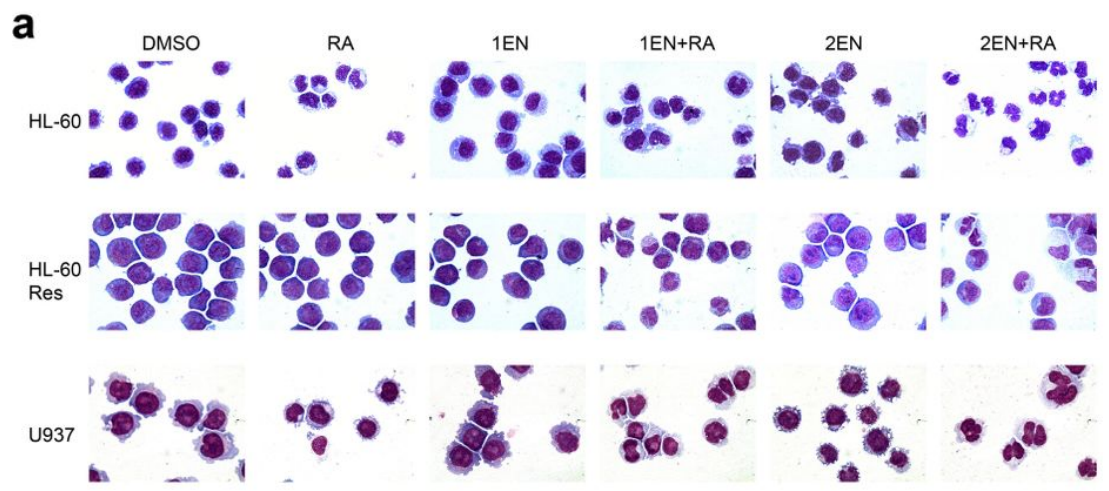

b

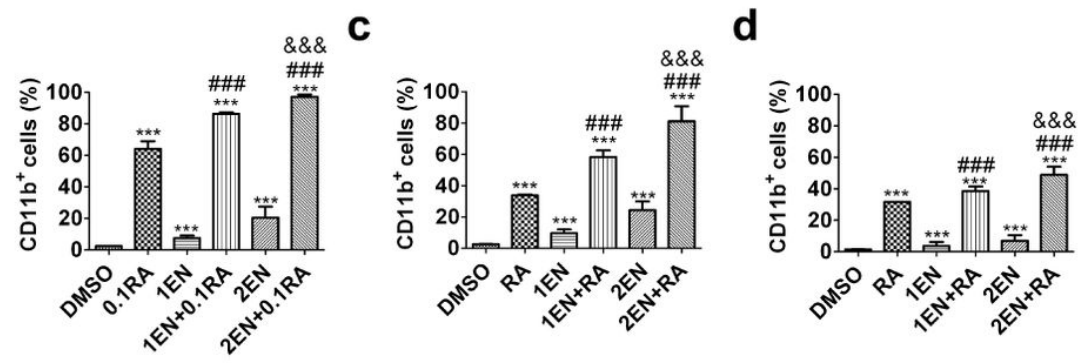

e

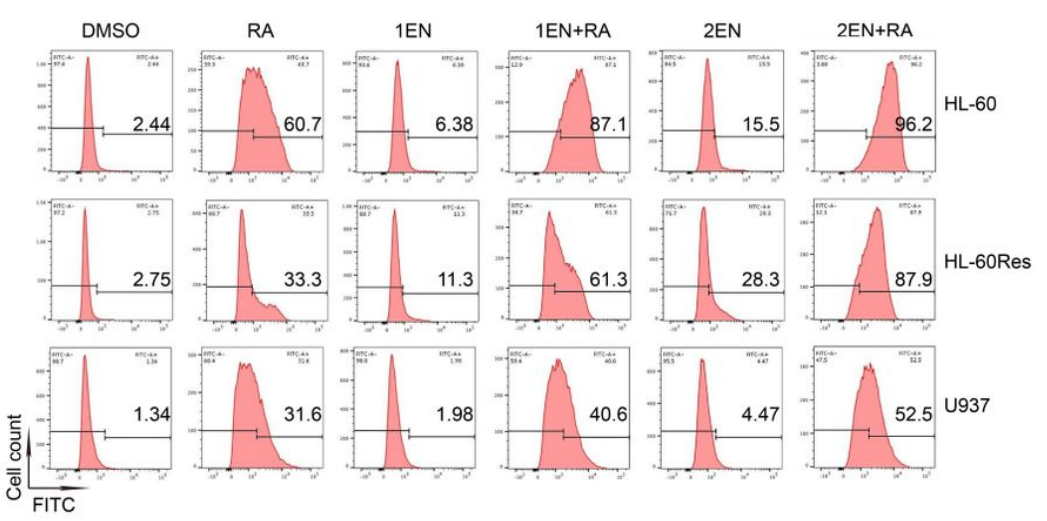

Figure 1

The effect of enz-ATRA treatment on cell differentiation in HL-60, HL-60Res and U937 cells. (a) HL-60 cells were treated with $1 / 2 \mu \mathrm{M}$ enzastaurin (1EN/2EN) and/or $0.1 \mu \mathrm{M}$ ATRA (RA) for 3 days. HL-60Res and U937 cells were treated with 1/2 $\mu \mathrm{M}$ enzastaurin (1EN/2EN) and/or $1 \mu \mathrm{M}$ ATRA (RA) for 10 days and 3 days, respectively. One representative morphology of HL-60 (upper panel), HL-60Res (middle panel) and U937 (lower panel) cells are shown. Magnification is 1,000. Similar results were obtained in three independent experiments. Differentiation was also evaluated by flow-cytometric analysis of CD11b expression in HL-60 (b), HL-60Res (c) and U937 cells (d) with the indicated treatment for 4 days, 6 days and 3 days, respectively. Each value represents the mean \pm SD of three independent measurements. ${ }^{\star *}{ }^{2}<<0.001$, versus DMSO treated cells. \#\#\# P<0.001, versus ATRA treated cells. \&\&\& $\mathrm{P}<0.001$, as compared with $1 \mathrm{EN}+\mathrm{RA}$ in U937 and HL-60Res cells or 1EN+0.1RA in HL-60 cells. (e) The representative histogram of flowcytometric analysis of CD11b expression in HL-60, HL-60Res and U937 cells with the indicated treatment for 4 days, 6 days and 3 days, respectively. The percentages of CD11b+ cells are shown in the corresponding panels. 
a

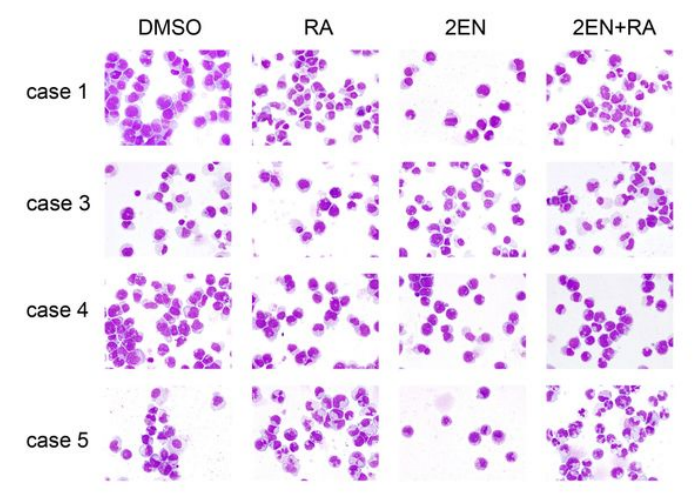

b
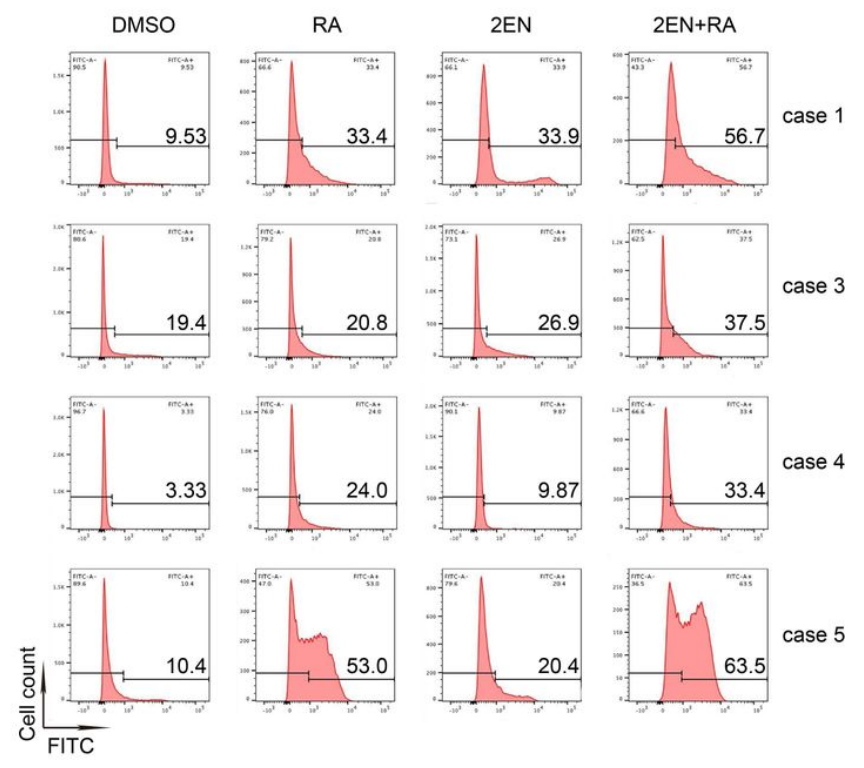

\section{Figure 2}

Enzastaurin enhances ATRA-induced differentiation in some primary blasts from non-APL AML patients. Primary blasts from patients were treated with $2 \mu \mathrm{M}$ enzastaurin (EN) and/or $1 \mu \mathrm{M}$ ATRA (RA) for 4 days. The morphology (a) and the histogram of flow-cytometric analysis of CD11b expression (b) are shown. Magnification of morphology is 1,000. The percentages of CD11b+cells are shown in the corresponding panels. 
a

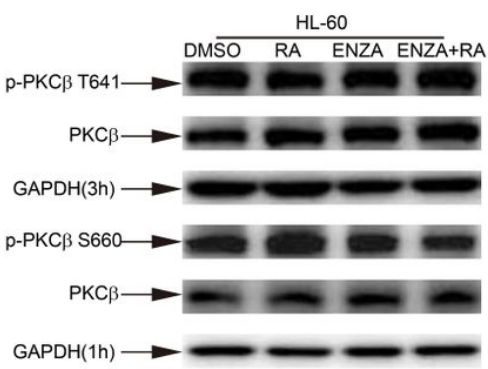

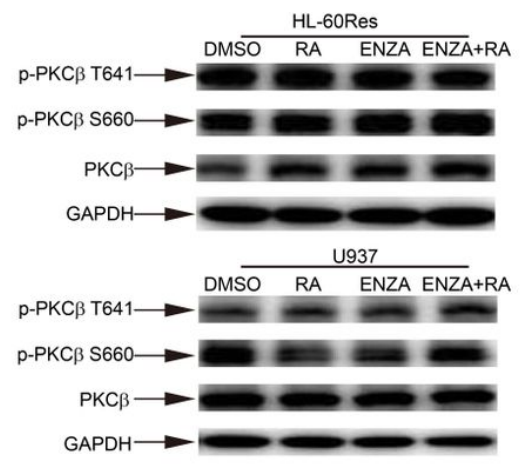

C

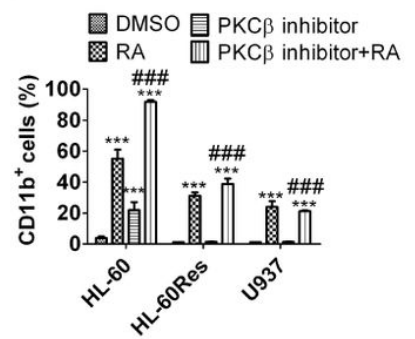

$\mathbf{e}$

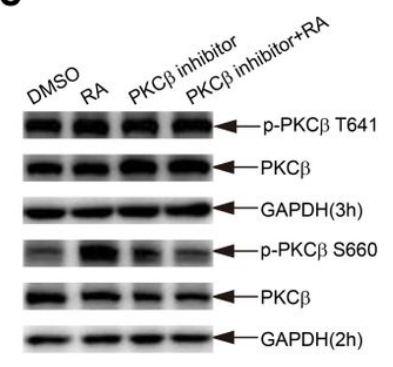

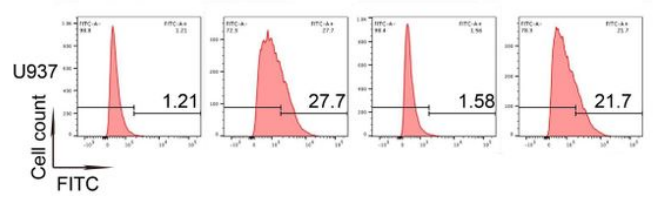

Figure 3

PKC $\beta$-inhibition mediates enzastaurin-enhanced ATRA-triggered differentiation of HL-60 cells. (a) HL-60 cells were treated with $2 \mu \mathrm{M}$ enzastaurin (EN) and/or $0.1 \mu \mathrm{M}$ ATRA (RA) for $1 \mathrm{~h}$ and $3 \mathrm{~h}$. HL-60Res and U937 cells were treated with $2 \mu \mathrm{M}$ enzastaurin (EN) and/or $1 \mu \mathrm{M}$ ATRA (RA) for $3 \mathrm{~h}$. The activation of $\mathrm{PKC} \beta$ was evaluated by Western-blotting analysis of phosphorylated PKC $\beta$ at serine 660 and threonine 641 . The same membrane incubated with anti-phospho-PKC $\beta$ was stripped and followed by detection of PKC $\beta$. Different time points for protein collections have corresponding GAPDH expression as internal control. (b) HL-60 cells were treated with $500 \mathrm{nM} \mathrm{PKC} \beta$ inhibitor and/or $0.1 \mu \mathrm{M}$ ATRA (RA) for 3 days. HL-60Res cells were treated with $200 \mathrm{nM}$ PKC $\beta$ inhibitor and/or $1 \mu \mathrm{M}$ ATRA (RA) for 10 days. U937 cells were treated with $100 \mathrm{nM}$ PKC $\beta$ inhibitor and/or $1 \mu \mathrm{M}$ ATRA (RA) for 3 days. The representative morphology of HL-60, HL-60Res and U937 cells are shown. Magnification is 1,000. (c) Differentiation was also evaluated by flow-cytometric analysis of CD11b expression in HL-60, HL-60Res and U937 cells with the indicated treatment for 4 days, 6 days and 3 days, respectively. Each value represents the mean \pm SD of three independent measurements. ${ }^{* * *} P<0.001$, versus DMSO treated cells. \#\#\# $P<0.001$, versus ATRA treated cells. (d) The representative histogram of flow-cytometric analysis of CD11b expression in HL-60, HL-60Res and U937 cells with the indicated treatment for 4 days, 6 days and 3 days, respectively. The percentages of CD11b+ cells are shown in the corresponding panels. (e) HL-60 cells were treated with $500 \mathrm{nM}$ PKC $\beta$ inhibitor and/or $0.1 \mu \mathrm{M}$ ATRA (RA) for $2 \mathrm{~h}$ and $3 \mathrm{~h}$. Phosphorylation of PKC $\beta$ was measured by Western-blotting analysis. The same membrane incubated with anti-phospho-PKC $\beta$ was stripped and followed by detection of PKC $\beta$. Different time points for protein collections have corresponding GAPDH expression as internal control. 


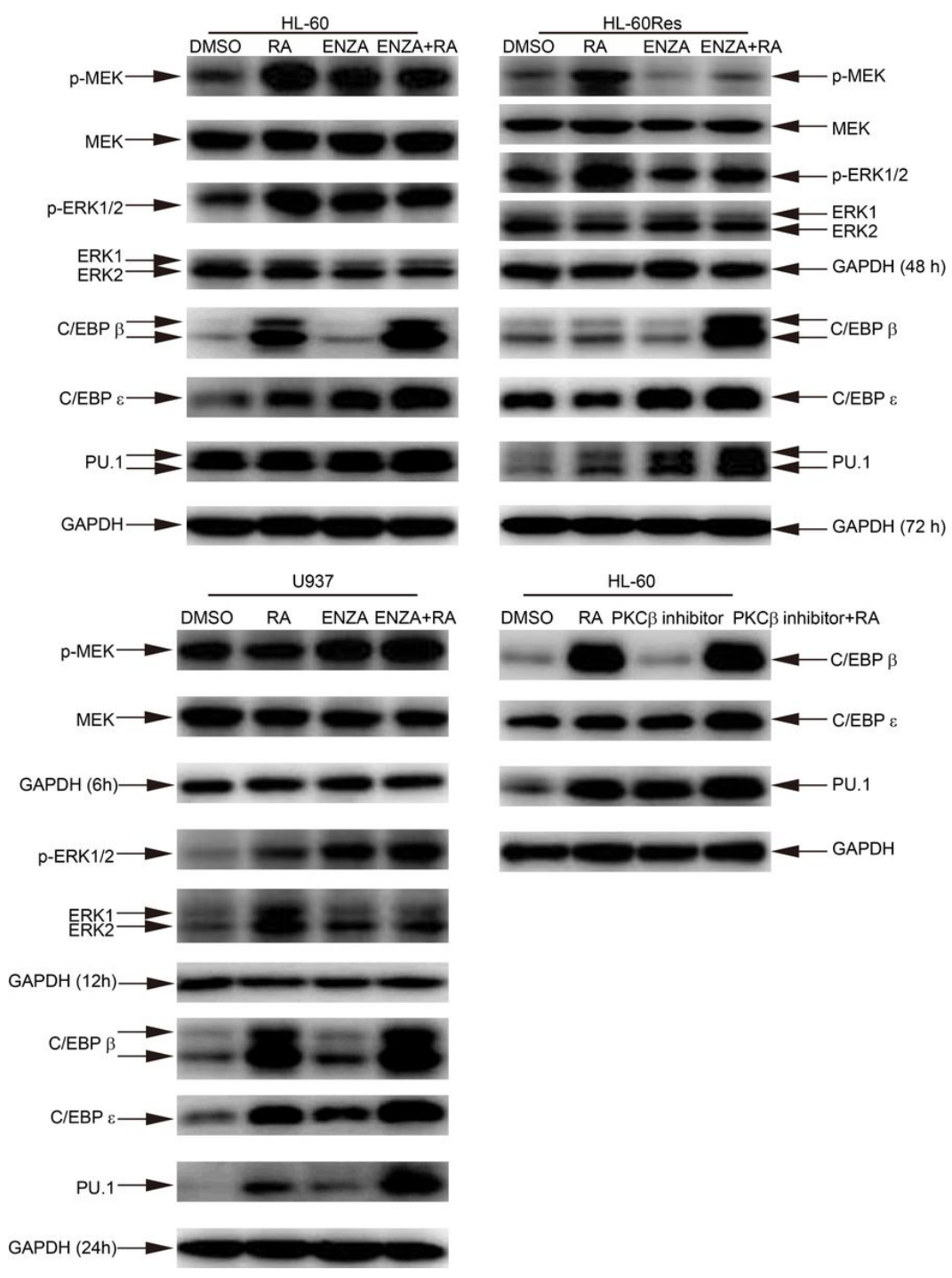

\section{Figure 4}

The effect of Enz-ATRA and PKC $\beta$ inhibitor-ATRA on C/EBPs, PU.1 and MEK/ERK. HL-60 cells (upper left) were treated with $2 \mu \mathrm{M}$ enzastaurin (EN) and/or $0.1 \mu \mathrm{M}$ ATRA (RA) for $24 \mathrm{~h}$. For PKC $\beta$ inhibitor, $500 \mathrm{nM}$ was used in HL-60 cells for $24 \mathrm{~h}$ (lower right). U937 cells (lower left) were treated with 2 $\mu \mathrm{M}$ enzastaurin (EN) and/or $1 \mu \mathrm{M}$ ATRA (RA) for $6 \mathrm{~h}, 12 \mathrm{~h}$ and $24 \mathrm{~h}$. HL-60Res (upper right) cells were treated with $2 \mu \mathrm{M}$ enzastaurin (EN) and/or $1 \mu \mathrm{M}$ ATRA (RA) for $48 \mathrm{~h}$ and $72 \mathrm{~h}$. The same membrane incubated with the antibodies to phosphorylated Erk $1 / 2$ or MEK $1 / 2$ was stripped and followed by detection of MEK and ERK1/2. Since diverse time points for collecting protein were used, each has the expression of GAPDH as internal control. Similar results were obtained in three independent experiments. 
b

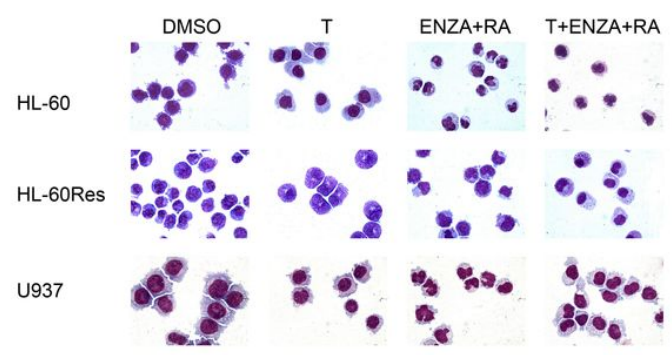

C

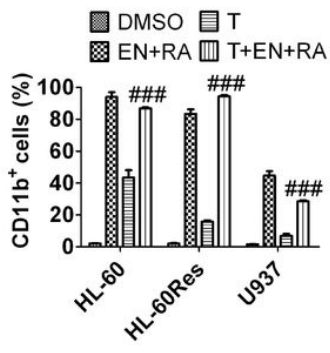

d

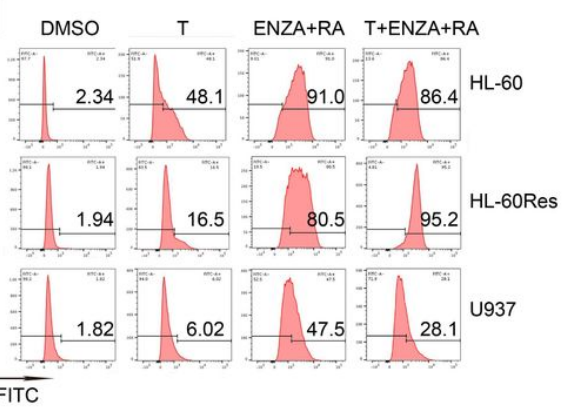

e

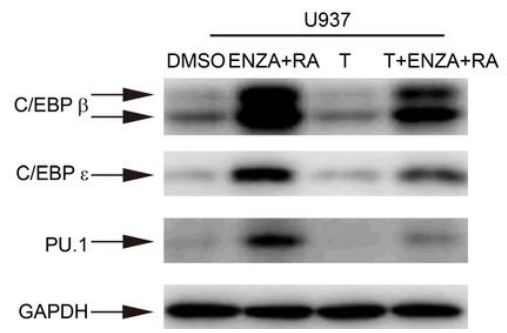

\section{Figure 5}

MEK/ERK-inhibition suppresses enz-ATRA-triggered differentiation of U937 cells by restoring the expression of C/EBPs and PU.1. HL-60, HL-60Res and U937 cells were pretreated with $0.01 \mu \mathrm{M}, 0.1 \mu \mathrm{M}$ and $5 \mu \mathrm{M}$ trametinib for $2 \mathrm{~h}$, respectively. (a) The attenuation of MEK activation by trametinib ( $\mathrm{T}$ ) was detected by Western-blotting analysis of phosphorylated ERK1/2 in HL-60, HL-60Res and U937 cells with indicated treatments for $24 \mathrm{~h}, 48 \mathrm{~h}$ and $4 \mathrm{~h}$, respectively. The same membrane incubated with the antibodies to phosphorylated Erk1/2 was stripped and followed by detection of ERK1/2. The expression of GAPDH was evaluated as internal control. Similar results were obtained in three independent experiments. (b) Effect of trametinib on morphology in HL-60, HL-60Res and U937 cells incubated with the indicated drugs for 4 days, 10 days and 3 days, respectively. The magnification is 1,000. One representative experiment among three independent assays is shown. Similar results were obtained in three independent experiments. (c) Differentiation was also evaluated by flow-cytometric analysis of CD11b expression in HL-60, HL-60Res and U937 cells with the indicated treatment for 4 days, 6 days and 3 days, respectively. Each value represents the mean \pm SD of three independent measurements. \#\#\# P<0.001, as compared with 2EN+RA in U937 and HL-60Res cells or 2EN+0.1RA in HL-60 cells. (d) The representative histogram of flow-cytometric analysis of CD11b expression in HL-60, HL-60Res and U937 cells with the indicated treatment for 4 days, 6 days and 3 days, respectively. The percentages of CD11b+ cells are shown in the corresponding panels. (e) The protein levels of C/EBP囚, C/EBP囚 and PU.1 in U937 cells with the indicated drugs for $24 \mathrm{~h}$ was assayed by Westernblotting. The expression of GAPDH was evaluated as internal control. Similar results were obtained in three independent experiments. 
a

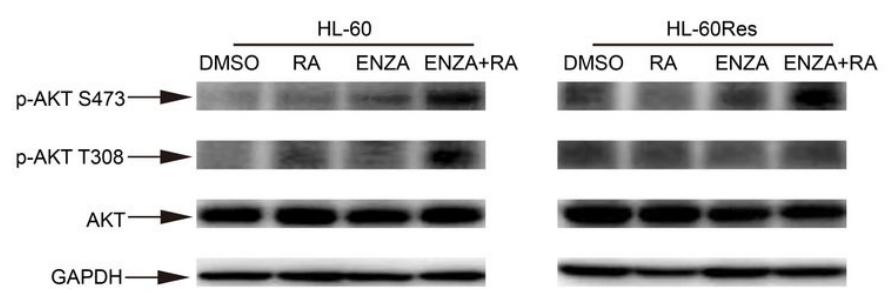

b
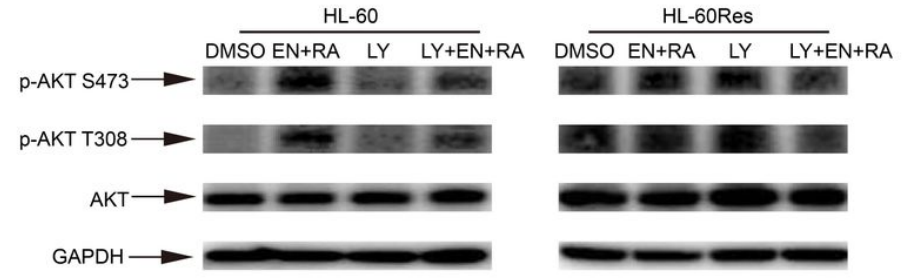

C

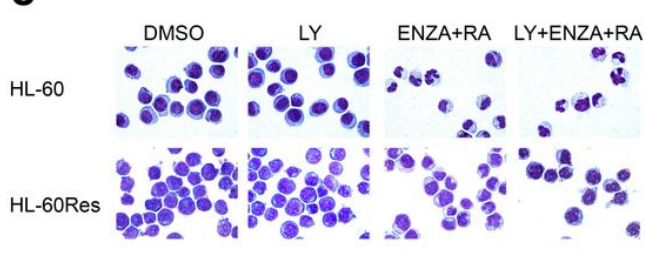

d $\square$ DMSO 目LY

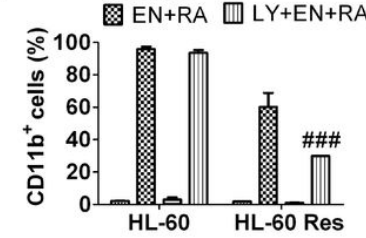

e

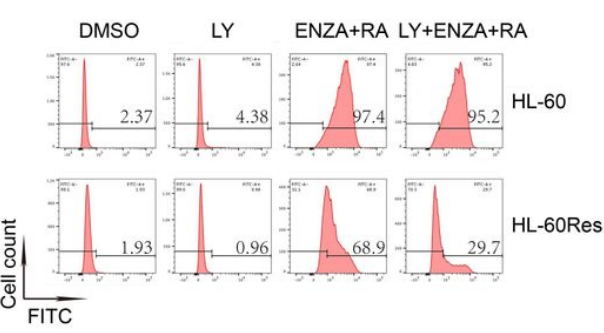

f

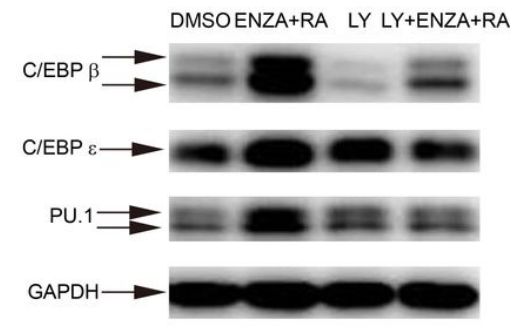

Figure 6

AKT controls enz-ATRA-induced differentiation of HL-60Res cells by enhancing the expression of C/EBPs and PU.1. (a) HL-60 cells were treated with 2 $\mu \mathrm{M}$ enzastaurin (EN) and/or $0.1 \mu \mathrm{M}$ ATRA (RA) for $48 \mathrm{~h}$. HL-60Res cells were treated with $2 \mu \mathrm{M}$ enzastaurin (EN) and/or $1 \mu \mathrm{M}$ ATRA (RA) for $72 \mathrm{~h}$. The activation of AKT was evaluated by Western-blotting analysis of phosphorylated AKT at serine 473 and threonine 308 . The same membrane incubated with anti-phospho-AKT was stripped and followed by detection of AKT. The expression of GAPDH was evaluated as internal control. (b) The attenuation of AKT activation by LY294002 (LY) was detected by Western-blotting analysis of phosphorylated AKT in HL-60 and HL-60Res cells with indicated treatments for $48 \mathrm{~h}$ and $72 \mathrm{~h}$, respectively. The same membrane incubated with the anti-phospho-AKT was stripped and followed by detection of AKT. The expression of GAPDH was evaluated as internal control. (c) The effect of LY294002 on morphology in HL-60 and HL-60Res cells incubated with the indicated drugs for 4 days and 10 days, respectively. The magnification is 1,000 . One representative experiment among three independent assays is shown. (d) Differentiation was also evaluated by flow-cytometric analysis of CD11b expression in HL-60 and HL-60Res cells with the indicated treatment for 4 days and 6 days, respectively. Each value represents the mean \pm SD of three independent measurements. \#\#\# $P<0.001$, as compared with 2EN+RA in HL-60Res cells. (e) The representative histogram of flow-cytometric analysis of CD11b expression in HL-60 and HL-60Res cells with the indicated treatment for 4 days and 6 days, respectively. The percentages of CD11b+cells are shown in the corresponding panels. (f) The protein levels of $\mathrm{C} / \mathrm{EBP} \otimes, \mathrm{C} / \mathrm{EBP} \otimes$ and PU. 1 in HL-60Res cells with the indicated drugs for $72 \mathrm{~h}$ was assayed by Western-blotting. The expression of GAPDH was evaluated as internal control.

\section{Supplementary Files}

This is a list of supplementary files associated with this preprint. Click to download.

- additionalfile1.tif 